\title{
Exact Solutions of Space Dependent Korteweg-de Vries Equation by the Extended Unified Method
}

\author{
Hamdy I. Abdel-Gawad ${ }^{1}$, Nasser S. Elazab ${ }^{2}$, Mohamed Osman $^{3}$ \\ Department of Mathematics, Faculty of Science, Cairo University, Giza-Egypt
}

Abstract: Recently the unified method for finding traveling wave solutions of non-linear evolution equations was proposed by one of the authors ${ }^{a}$. It was shown that, this method unifies all the methods being used to find these solutions. In this paper, we extend this method to find a class of formal exact solutions to Korteweg-de Vries $(K d V)$ equation with space dependent coefficients. A new class of multiple-soliton or wave trains is obtained.

Keywords: Exact solution, Extended unified method, Korteweg-deVries equation, variable coefficients

We consider the following evolution equation

\section{Introduction}

$$
f\left(x, t, u, \frac{\partial u}{\partial t}, \frac{\partial u}{\partial x}, \frac{\partial^{2} u}{\partial x \partial t}, \frac{\partial^{2} u}{\partial x^{2}}, \ldots, \frac{\partial^{m} u}{\partial x^{m}}\right)=0, \quad m \geq 1,
$$

where $f$ is a polynomial in its arguments. When Eq. (1) does not depend explicitly on $x$ and $t$, it can be reduced to a subclass of ordinary differential equations by using the Lie groups for partial differential equations [1] or by using similarity transformations. Among these equations, the traveling wave has the form

$$
g\left(u, u^{\prime}, u^{\prime \prime}, \ldots u^{(m)}\right)=0, u^{\prime}=\frac{d u}{d z}, z=x-c t,
$$

which results due to the translation symmetry of (1). The Painleve' analysis is used to testing the integrability of partial differential equations, that was developed in [2]. Auto-B $\ddot{a}$ cklund transformation deals with the exact solutions that were obtained for integrable forms of (2) by truncating Painleve' expansion [3-9]. Recently autoB $\ddot{a}$ cklund transformation that was extrapolated in [10-14] and the homogeneous balance method in [15-19] assert a solution for evolution equations with variable coefficients in the form

$$
u(x, t)=\frac{\partial^{m-2}}{\partial x^{m-2}}\left(a(\phi) \phi_{x}\right)+u^{(0)}(x, t),
$$

where $\phi$ is the base function.

\section{Extended unified method}

Explicit solutions of evolution equations of type (2) are, in fact, particular solutions. In this respect, these solutions are mapped to other solutions that are given in terms of known elementary or special functions. Recently in [20] the class of these solutions was obtained by the generalized mapping method (GMM). This method generalizes the results as polynomial or rational function solutions. In the present paper, we extend this method to handle equations of type (1).

\section{1- Polynomial Solutions}

In this section, we search for polynomial solutions of equations (2) in

$$
\begin{gathered}
C^{S}(\mathrm{R}), \quad S=\left\{\phi: R \times R^{+} \rightarrow K, \phi_{t}^{q}=P_{k_{1}}^{t}(\phi),\left(\phi_{x}\right)^{p}=P_{k}^{x}(\phi)\right\}, \\
P_{k_{1}}^{t}(\phi)=\sum_{i=0}^{k_{1}} b_{i}(x, t) \phi^{i}(x, t), \quad P_{k}^{x}(\phi)=\sum_{i=0}^{s} c_{i}(x, t) \phi^{i}(x, t) .
\end{gathered}
$$

Indeed the set $S$ contains elementary or elliptic functions for some particular values of $q, p, k$ and $k_{1}$. The mapping method asserts that there exists a positive integer $n$ and a mapping

$$
M: C^{S}(R) \rightarrow \Omega, \quad \Omega=\left\{v, v=\sum_{i=0}^{s} a_{i}(x, t) \phi^{i}(x, t), \phi \in S\right\}
$$


Such that $M(u)=P_{n}(\phi)$ and satisfies the properties

$$
\begin{gathered}
M\left(\alpha_{1} u_{1}+\alpha_{2} u_{2}\right)=\alpha_{1} M\left(u_{1}\right)+\alpha_{2} M\left(u_{2}\right), \\
M\left(u_{1} u_{2}\right)=M\left(u_{1}\right) M\left(u_{2}\right), M\left(u_{t}\right)=(M(u))_{t}, M\left(u_{x}\right)=(M(u))_{x} .
\end{gathered}
$$

Thus $M$ is a ring homomorphism that conserves differentiation. By the former conditions, we find that

$$
M\left(u_{t}\right)=P_{\left(n-1+k_{1}\right.}^{t}(\phi) \in \Omega, \quad M\left(u_{x}\right)=P_{(n-1+k)}^{x}(\phi) \in \Omega
$$

By using the properties of $M$ and the last results and as $f=f\left(x, t, u, u_{t}, \ldots ..\right)$ is a polynomial in its arguments, we find that $M(f)$ is a polynomial and there exists $s_{0} \leq s$ such that $M(f)=P_{s_{0}}(\phi) \in \Omega$. It is worthy to notice that all these polynomials have different coefficients. More simply the mapping $M$ assigns to $u$ and $f$ gives two auxiliary equations, the polynomials $P_{n}(\phi)$ and $P_{s_{0}}(\phi)$ respectively. In case of equations (1) $s_{0}=n-m+m k$. The utility of the above presentation helps us to give arguments to the statements of the conditions in lemmas 2.1 and 2.2. Also, we think that it allows for constructing more generalization and it is more appropriate when (1) is a vector equation.

We substitute for $u, u_{t}, u_{x}, \ldots ., \frac{\partial^{m} u}{\partial x^{m}}$ as polynomials in $\phi$, so that the function $f$ is a polynomial in $\phi$, together with two auxiliary equations. In the applications we may write directly $u=P_{n}(\phi), f=P_{s_{0}}(\phi)$.

From the previous analysis we may write

$$
u=\sum_{i=0}^{n} a_{i}(x, t) \phi^{i},
$$

where for instance we assume that $k_{1}=k$, so that the auxiliary equations are

$$
\phi_{t}=\sum_{i=0}^{k} b_{i}(x, t) \phi^{i}, \phi_{x}=\sum_{i=0}^{k} c_{i}(x, t) \phi^{i},
$$

together with the compatibility equation

$$
\phi_{x t}=\phi_{t x} .
$$

We mention that solutions of (4) when exist, are elementary ( $p=q=1$ ). The case of elliptic solutions ( $p=q=2$ ) will be considered in a future work.

When substituting from (3) and (4) into (1) we find that it is transformed to $P_{s_{o}}^{(f)}(\phi) \equiv 0$ that gives rise to

$$
\begin{gathered}
\sum_{i=0}^{s_{o}} h_{i}\left(a_{r_{o}}(x, t), b_{r_{1}}(x, t), c_{r_{2}}(x, t), a_{r_{o} t}(x, t), a_{r_{o} x}(x, t), \ldots . .\right) \phi^{i} \equiv 0, \\
r_{0}=0,1, \ldots, n \quad \text { and } \quad r_{1}, r_{2}=0, \ldots, k .
\end{gathered}
$$

By equating the coefficients of $\phi^{i}, i=0,1, \ldots, s_{o}$ to zero, we get a set of $\left(s_{o}+1\right)$ algebraic (or differential) equations, namely the principle equations, in the functions $a_{i}, b_{i}$ and $c_{i}$. On the other hand the equations that result from (5) count: $2 k-1, k \geq 2$. We mention that these later unknown functions count: $n+2 k+3$.

In Eq.(1), if $u^{j} u_{x}$ and $\frac{\partial^{m}}{\partial x^{m}} u$ are the highest nonlinear and the highest order derivative terms respectively, then we get the balancing condition as

$s_{o}=n j+n+k-1=n-m+m k$. Thus by solving for $n$, we find that it depends on $m, j$ and $k$.

The last result and the number of compatibility equations namely $2 k-1, k \geq 2$ determine if the equations to be solved are over-determined or under-determined. The number of the determining equations, balances the number of unknowns, is over-determined or is under-determined when the difference, namely $(n-m+m k+1)+(2 k-1)-(n+2 k+3)$ is $0,>0$, or $<0$ respectively. From this last conditions, we may determine a consistency condition that will be identified in the lemmas. In what follows necessary conditions for the existence of polynomial solutions will be stated. 
Lemma 2.1. For polynomial-solutions of (1) (as a polynomial in $\phi$ ) to exist it is necessary that

(i) $(m-1)(k-1) / j(:=n)$ is a positive integer

(ii) $m(k-1)-3 \leq m$ when the equation (1) in the absence of $x$, and $t$ passes the Painleve' test . Otherwise $m$ is replaced by 2.

We notice that the first and the second conditions in lemma 2.1 are the balancing and the consistency conditions respectively. For details see [20].

\section{2 - The rational function solutions}

Here, also we search for solution of equation (1) in $C^{S}(R)$. For rational function solutions of equation (1), we consider the space of functions

$\Omega_{R}=\left\{v, v=P_{n}(\phi) / Q_{r}(\phi), \phi \in S\right\}$ and $Q_{r}(\phi)$ has no zeros in $K \subset R$. The definitions in the above and the GMM for rational function solutions assert that there exists a mapping

$$
M_{R}: C^{S}(R) \rightarrow \Omega_{R}, \quad M_{R}(u)=P_{n}(\phi) / Q_{r}(\phi), \quad \phi \in S .
$$

The properties of these mapping are the same properties of the mapping $M(u)$ in section 2.1. By bearing in mind these properties and from equations (4) and (5), we find that

$$
M_{R}\left(u_{t}\right)=P_{1,(n-1+k+r)}(\phi) / Q_{r}^{2}(\phi), \quad M_{R}\left(u_{x}\right)=P_{2,(n-1+k+r)}(\phi) / Q_{r}^{m+1}(\phi),
$$

Thus, $M_{R}\left(\frac{\partial^{i} u}{\partial x^{i}}\right) \in \Omega_{R}, \quad i=1,2, \ldots, m$. By using the properties of $M_{R}$ and the last results, we get $M_{R}(f) \in \Omega_{R}$ and there exist $s_{1} \leq s$ such that $M_{R}(f)=P_{s_{1} R}(\phi) / Q_{r}^{m+1}(\phi)$. Indeed $s_{1}$ depends on $n, r, k, m$, where in the case mentioned in the above $s_{1}=n-m+m k+m r$. Simply, we write

$$
u=\sum_{i=0}^{n} a_{i} \phi^{i} / \sum_{i=0}^{r} d_{i} \phi^{i}
$$

So that the Eq. (1) is transformed to $P_{s_{1} R}(\phi) \equiv 0$. Equivalently, the last identity becomes

$$
\begin{gathered}
\sum_{i=0}^{s_{1}} h_{i}\left(a_{r_{0}}(x, t), d_{r_{2}}(x, t), b_{r_{1}}(x, t), c_{r_{3}}(x, t), \ldots .\right) \phi^{i} \equiv 0, \\
r_{0}=0, \ldots, n, r_{1}, r_{3}=0, \ldots, k, \quad \text { and } \quad r_{2}=0, \ldots, r .
\end{gathered}
$$

In (8), by equating the coefficients of $\phi^{i}, i=0,1, \ldots, s_{1}$ to zero, we get a set of $\left(s_{1}+1\right)$ equations that determine the functions $a_{i}, b_{i}, c_{i}$ and $d_{i}$. We mention that these later functions count $n+2 k+r+3$. By using the same assumptions on Eq. (1), as in section 2.1, the balancing condition is

$$
\left\{\begin{array}{cc}
n j+n+k-1+r=n-m+m k+m r+r(j-(m+1)), & m+1<j \\
n j+r((m+1)-j)=n-m+m k+m r=s_{1}, & m+1>j
\end{array}\right.
$$

Now by solving (9) for $n$, we find that it depends on $m, j, r$ and $k$ and, in both two cases, we get the same equation for $n-r$. Hereafter, we distinguish between the two cases mentioned in (9). From the last results and when $j<m+1$, the number of the determining equations, balances the number of unknowns, is overdetermined or is under-determined when the difference, namely

$(n-m+m k+r m+1)+(2 k-1)-(n+2 k+r+3)$ is $0,>0$, or $<0$ respectively.

But when $j>m+1$ this difference is

$(n-m+m k+r m+1+r(j-(m+1)))+(2 k-1)-(n+2 k+r+3)$. From these last conditions, we may determine the consistency condition that will be identified in the following Lemma.

Lemma 2.2. For solitary wave-rational solutions of Eq. (2) to exist it is necessary that

(i) $(m-1)(k-1) / j(:=n-r)$ is an integer

(ii) $r(m-1)+(k-1) m-3 \leq m, j<m+1$ or

$r(j-2)+(k-1) m-k-2 \leq 2, j>m+1$, in the case when Eq. (1) passes the Painleve' test. Otherwise $r(m-1)+(k-1) m-k-2 \leq 2, j<m+1$ or $r(j-2)+(k-1) m-k-2 \leq 2, j>m+1$. 
For details see [20].

\section{Exact solutions of space dependent $\mathrm{KdV}$ equation}

Here, we extend the unified method to the variable coefficient KdV equation

(10) $u_{t}+f(x) u_{x x x}+g(x) u u_{x}=0, t>0, x>0$,

where $f$ and $g$ are arbitrary functions in $x$. For $x<0$, the solutions of Eq. (10) hold if we replace $x$ by $|x|$ and assuming that $f(-x)=-f(x)$ and $g(-x)=-g(x)$. We mention that Eq. (10) describes the propagation of waves in a medium with space dependent dispersion and conviction. In fact, differential equations with variable coefficients may be of practical interests. Some exact solutions were obtained in Nirmala and Vedan [21] and E. Fan [12] when the coefficients in Eq. (10) are time dependent, namely $f(t)$ and $g(t)$. In these works, solutions were obtained when $f(t)=c g(t)$, where $c$ is a constant.

\subsection{The polynomial function solutions}

In what follows we shall derive a polynomial solution of equation Eq. (10In lemma 2.1, the consistency condition holds when $k=2,3$ but it does not hold when $k \geq 4$. So that, only the cases $k=2,3$ will be considered. When $k=2, n=2$, by substituting into (3), (4) and (10), we get six principle equations. We mention that calculations are carried out by using MATHEMATICA where standard functions in calculus and algebra were only needed. The steps of computations are as follows;

Step 1. Solving the principle equations, where five of them are solved explicitly to

$$
\begin{gathered}
a_{2}(x, t)=-12 h(x) c_{2}(x, t)^{2}, \\
a_{1}(x, t)=-\frac{12}{5}\left(5 c_{1}(x, t) c_{2}(x, t) h(x)+c_{2}(x, t) h^{\prime}(x)+5 h(x) c_{2 x}(x, t)\right), \\
a_{2}(x, t)=-12 h(x) c_{2}(x, t)^{2}, \\
a_{1}(x, t)=-\frac{12}{5}\left(5 c_{1}(x, t) c_{2}(x, t) h(x)+c_{2}(x, t) h^{\prime}(x)+5 h(x) c_{2 x}(x, t)\right),
\end{gathered}
$$

together with explicit equations for $b_{2}(x, t), b_{1}(x, t)$ and $b_{0}(x, t)$ (they are too lengthy to written here) where $h(x)=\frac{f(x)}{g(x)}$ and we put $r(x)=\frac{1}{g(x)}$. It remains only one unsolved equation of the principle ones.

Step 2. We consider the compatibility equations that result from $\phi_{x t}=\phi_{t x}$ and they are given formally by;

$$
\begin{gathered}
b_{0}(x, t) c_{1}(x, t)-b_{1}(x, t) c_{0}(x, t)+c_{0 t}(x, t)-b_{0 x}(x, t)=0, \\
2 b_{0}(x, t) c_{2}(x, t)-2 b_{2}(x, t) c_{0}(x, t)+c_{1 t}(x, t)-b_{1 x}(x, t)=0, \\
-b_{2}(x, t) c_{1}(x, t)+b_{1}(x, t) c_{2}(x, t)+c_{2 t}(x, t)-b_{2 x}(x, t)=0 .
\end{gathered}
$$

To simplify the computations, we make the transformations

$$
\begin{gathered}
c_{2 x}(x, t)=p(x, t) c_{2}(x, t), c_{1}(x, t)=-p(x, t)+C_{1}(x, t), \\
c_{0}(x, t)=\frac{-2 C_{1 x}(x, t)+C_{1}^{2}(x, t)+4 C_{0}(x, t)}{4 c_{2}(x, t)},
\end{gathered}
$$

where $C_{0}(x, t), C_{1}(x, t)$ are arbitrary functions. To evaluate $a_{0}(x, t)$ the following steps are used.

i- Solve the last equation in (12) for $a_{0 x}$

ii- Eliminate $a_{0 x x}, a_{0 x x x}$

iii- Substitute in the middle equation in (12) to get $a_{0}(x, t)$

iv- Calculate $a_{0 x}$ from the last step and identify it by $a_{0 x}$ from step (i), we get an equation in $C_{0}, C_{0 x}, \ldots$.

As the computations are too lengthy in the general case, we consider a power law functions 
$h(x)=h_{0} x^{n}, r(x)=r_{0} x^{m}$. In the original variable $g(x)=r_{0}^{-1} x^{-m}, f(x)=\frac{h_{0}}{r_{0}} x^{n-m}$.

v- Solve the equation that result from (iv) in $C_{0 x}$

vi- Substitute into the first equation in (12) and solve for $C_{0 t}$. Thus (12) solved completely.

vii- Calculate $C_{0 t x}$ from (vi) and balance it with $C_{0 x t}$ from (v), we get the following algebraic equations

$$
30+5 m^{2}+m(25-9 n)-22 n+4 n^{2}=0,
$$

or

$-187500 m+31250 m^{2}+125000 m^{3}+31250 m^{4}-150000 n+893750 m n+84375 m^{2} n$ $-362500 m^{3} n-90625{ }^{4} n+695000 n^{2}-1605000 m n^{2}-477500 m^{2} n^{2}+325000 m^{3} n^{2}+$ $81250 m^{4} n^{2}-1274000 n^{3}+1338500 m n^{3}+581750 m^{2} n^{3}-87500 m^{3} n^{3}-21875 m^{4} n^{3}+$

$1178000 n^{4}-511500 m n^{4}-255750 m^{2} n^{4}-578000 n^{5}+71750 m n^{5}+35875 m^{2} n^{5}+$

$$
143000 n^{6}-14000 n^{7}+5(5-n) \sqrt{W(m, n)}=0,
$$

Where

$$
W(m, n)=(2-n)\left(5 m^{2}+4(-1+n) n+m(-5+9 n)\right)^{2}\left(\left(-450000 m^{5}\left(5-8 n+3 n^{2}\right)+25 m^{4} \times\right.\right.
$$

$\left(-209750+499175 n-270640 n^{2}+24307 n^{3}\right)+10 m^{3}(-788750+1335125 n+328025$

$\left.\times n^{2}-939245 n^{3}+237213 n^{4}\right)+m^{2}\left(9381250-53065625 n+94436500 n^{2}-68148950 n^{3}\right.$

$\left.-1899953 n^{5}\right)+4(n-1)^{2}\left(1406250+1621875 n-6959000 n^{2}\right)+5762450 n^{3}-1866010 n^{4}$

$\left.+214003 n^{5}\right)-4 m\left(-5718750+21213125 n-27985375 n^{2}+14726550 n^{3}+1320440 n^{4}\right.$

$$
\left.\left.-1178459 n^{5}+263349 n^{6}\right)\right) \text {. }
$$

The solution of Eq. (14) leads to $n=m+3$ or $n=\frac{5(2+m)}{4}$.

In what follows we find the solution of Eq. (10):

Case (1): when $n=m+3$, Eq. (15) leads to $m=2, \frac{16}{3},-1$.

First when $m=2$, we get $h(x)=h_{0} x^{5}, \quad r(x)=r_{0} x^{2}$ and in the the original variable $f(x)=\frac{h_{0}}{r_{0}} x^{3}, g(x)=\frac{1}{r_{0} x^{2}}$, by solving the first auxiliary equation in (4) (we get $\phi(x, t)$ ) and substituting into second auxiliary equation in (4) to find the arbitrary time dependent function of integration, we get $C_{1 t}(x, t)=0$, or $C_{1}(x, t)=C_{1}(x)$ and so, we get $\phi(x, t)$ as

$$
\phi(x, t)=\frac{2+\left(x-e^{\frac{10 h_{0} t}{r_{0}}}\right) C_{1}(x)}{2\left(e^{\frac{10 h_{0} t}{r_{0}}}-x\right) c_{2}(x, t)} .
$$

By substituting from (17), into (3) we get a solution of (10) as

$$
u(x, t)=-\frac{2 h_{0} x^{3}\left(x^{2}+e^{\frac{20 h_{0} t}{r_{0}}}+4 x e^{\frac{10 h_{0} t}{r_{0}}}\right)}{\left(-e^{\frac{10 h_{0} t}{r_{0}}}+x\right)^{2}} .
$$

It is worth noticing that one can verify that the solution given by (18) satisfies (10).

When $m=\frac{16}{13}$ in a way similar to the above, we get $h(x)=h_{0} x^{\frac{55}{13}}, r(x)=r_{0} x^{\frac{16}{13}}$ and in the the original variable $f(x)=\frac{h_{0}}{r_{0}} x^{3}, g(x)=\frac{1}{r_{0} x^{\frac{16}{13}}}$, also the auxiliary equations (3) solve to 


$$
\phi(x, t)=-\frac{22 e^{\frac{231 h_{0} t}{2197 r_{0}}}+195 x^{\frac{2}{13}}+26 e^{\frac{231 \alpha_{0} t}{2197_{0}}} x C_{1}(x, t)+169 x^{\frac{15}{13}} C_{1}(x, t)}{52 e^{\frac{231 h_{0} t}{2197 r_{0}}} x c_{2}(x, t)+338 x^{\frac{15}{13}} c_{2}(x, t)},
$$

and we get the solution of Eq. (10) as

$$
u(x, t)=-\frac{48 h_{0} x^{\frac{33}{13}}}{\left(2 e^{\frac{2310 h_{0} t}{2197 r_{0}}}+13 x^{\frac{2}{13}}\right)^{2}} .
$$

When $m=-1$, we get the following results

$$
\begin{aligned}
& \phi(x, t)=-\frac{6 e^{\frac{3 h_{0}}{5 r_{0}} t}+40 x^{\frac{3}{5}}+15 e^{\frac{3 h_{0}}{5 r_{0}} t} x C_{1}(x, t)+25 x^{\frac{8}{5}} C_{1}(x, t)}{30 e^{\frac{3 h_{0}}{5 r_{0}} t}+50 x^{\frac{8}{5}} C_{1}(x, t)}, \\
& u(x, t)=\frac{h_{0}\left(261 e^{\frac{6 h_{0}}{5 r_{0}} t}+870 e^{\frac{3 h_{0}}{5 r_{0}} t} x^{\frac{3}{5}}-1975 x^{\frac{6}{5}}\right)}{25\left(3 e^{\frac{3 h_{0}}{5 r_{0}} t}+5 x^{\frac{3}{5}}\right)^{2}}
\end{aligned}
$$

Case (2): when $n=\frac{5(2+m)}{4}$, Eq. (15) leads to $m=\frac{2}{3}$ and we get the following results

$$
\begin{gathered}
\phi(x, t)=-\frac{\left(1-\frac{28 h_{0} t}{9 r_{0}}\right)\left(2+3 x C_{1}(x, t)+12 x^{\frac{1}{3}}+9 x^{\frac{4}{3}} C_{1}(x, t)\right.}{6\left(1-\frac{28 h_{0} t}{9 r_{0}}\right) x c_{2}(x, t)+18 x^{\frac{4}{3}} c_{2}(x, t)}, \\
u(x, t)=-\frac{972 h_{0} r_{0}^{2} x^{2}}{\left(28 h_{0} t-9 r_{0}\left(1+3 x^{\frac{1}{3}}\right)\right)^{2}} .
\end{gathered}
$$

Again, the solutions (20) or (22) or (24) verify Eq. (10).

\section{Conclusions}

In this paper, we suggested an extended unified method for finding exact solutions to evolution equations with variable coefficients. A wide class of exact solutions to KdV equation with Space dependent coefficients is obtained. The method and the solutions that we obtained here are completely new and we can use this method to find exact solutions of coupled evolution equations. But in this case we think that parallel computations should be used.

\section{References}

[1] P. J. Olivier, Application of Lie Groups to Differential Equations. GTM, Vol. 107 (Berlin, Springer) (1986).

[2] J. Weiss, M. Tabor, G. Carenville, J. Math. Phys., 24, 522 (1983).

[3] R. Conte, Phys. Lett. A., 134, 100-104 (1988).

[4] B. Y. Gou and Z. X. Chen, J. Phys. A Math. Gen., 24, 645-650 (1991).

[5] H.I. Abdel-Gawad, J. Statis. Phys., 97, 395-407 (1999).

[6] C. Rogers and W. F. Shadwick, Bäcklund Transformations (Academic, New York) (1982).

[7] K. M. Tamizhmani and M. Lakshamanan, J. Phys. A, Math. Gen. , 16 , 3773 (1983).

[8] Y. Xie, J. Phys. A Math. Gen., 375229 (2004).

[9] C. Rogers and Szereszewski, J. Phys. A Math. Theor. 42, 40-4015 (2009).

[10] E. Fan, and H. Zhang, Phys. Lett. A 245, 389-392 (1999)

[11] E. Fan, Phys. Lett. A,265, 353-257 (2000).

[12] E. Fan, Phys. Lett. A,294, 26-29 (2002).

[13] L. Yang, Z. Zhu, and Y. Wang, Phys. Lett A, 260, 55-59 (1999).

[14] E. M. H. Moussa, R. M. El-Shikh, Phys. Lett. A, 372, 1429-1431 (2008).

[15] M. L. Wang, and Y. B. zhou Phys. Lett. A 318, 84 (2003).

[16] M. L. Wang, and X. Z. Li, Phys. Lett. A, 343, 48 (2005).

[17] G. L. Cai, Q. C. Wang and J. J. Huang, Int. J. Nonl. Sci.2, 122-128 (2006).

[18] N. A. Kurdashov, Phys. Lett. A, 147, 287 (1990)

[19] M. Wang, J. Zhang, and X. Li, Phys. Lett. A,372, 417 (2008).

[20] H.I. Abdel-Gawad, J.Stat. Phys., 147, 506-518 (2012).

[21] N. Nirmala; M.J. Vedan; B.V. Baby; J. Math. Phys, Issue 11, 2640- 2643 (1986). 